\title{
Correlações em séries temporais de preços de manga produzida no Vale do São
}

\section{Francisco}

\author{
Correlations in time series of prices of mango produced in the São Francisco Valley \\ Correlaciones en series de tiempo de los precios del mango producido en el Valle de São Francisco
}

Recebido: 10/09/2021 | Revisado: 16/09/2021 | Aceito: 18/09/2021 | Publicado: 19/09/2021

Bruno de Freitas Assunção

ORCID: https://orcid.org/0000-0002-2045-884X

Universidade Federal Rural de Pernambuco, Brasil

E-mail: bruno071098@gmail.com

Ikaro Daniel de Carvalho Barreto

ORCID: https://orcid.org/0000-0001-7253-806X Universidade Federal Rural de Pernambuco, Brasil E-mail: daniel.carvalho.ib@gmail.com

Tatijana Stosic

ORCID: https://orcid.org/0000-0002-5691-945X Universidade Federal Rural de Pernambuco, Brasil E-mail: tastosic@gmail.com

Borko Stosic

ORCID: https://orcid.org/0000-0001-5031-6968 Universidade Federal Rural de Pernambuco, Brasil E-mail: borkostosic@gmail.com

\begin{abstract}
Resumo
Nas últimas décadas o comércio de frutas no Brasil cresceu expressivamente atendendo ao mercado interno e externo. Dentre as principais frutas produzidas e comercializadas, destaca-se a manga, a fruta mais exportada pelo Brasil. Neste trabalho foram analisadas as séries temporais de retornos e de volatilidade de preços semanais de duas variedades de manga, Palmer e Tommy Atkins, produzidas no Vale do São Francisco, a região com a maior produção de manga no país. Foram utilizados os métodos Detrended Fluctuation Analysis (DFA) e Detrended Cross Correlation Analysis (DCCA) para calcular expoentes de escala de autocorrelações e correlações cruzadas entre as séries analisadas. Os resultados mostraram que as séries de volatilidade apresentam persistência mais forte do que as séries de retornos que apresentaram dois regimes de invariância de escala com correlações antipersistentes nas escalas maiores. As correlações cruzadas entre as séries de retornos também apresentaram dois regimes de escala com expoentes semelhantes as séries de retornos da variedade Tommy. Os valores do coeficiente de correlação obtidos pelo método Detrended Cross Correlation Coefficient mostraram que para ambas, retornos e volatilidade, as correlações entre as séries são positivas, aumentam com escala temporal e são mais fortes para as séries de retornos.
\end{abstract}

Palavras-chave: Manga Tommy, Palmer; Correlações; Detrended fluctuation analysis; Detrended cross correlation coefficient.

\begin{abstract}
In recent decades, the fruit trade in Brazil has grown significantly, serving the domestic and foreign markets. Among the main fruits produced and traded, mango, the most exported fruit in Brazil, stands out. In this work we analyzed the time series of returns and volatility of weekly prices of two mango varieties, Palmer and Tommy Atkins produced in the São Francisco Valley, the region with the highest mango production in the country. The methods Detrended fluctuation analysis (DFA) and Detrended cross correlation analysis (DCCA) were used to calculate scaling exponents of autocorrelations and cross correlations between the analyzed series. The results showed that the volatility series have stronger persistence than the return series that presented two regimes of scale invariance with anti-persistent correlations on the larger temporal scales. Cross-correlations between the series of returns also show two scaling regimes with exponents similar to the series of returns of the Tommy variety. The values of the correlation coefficient obtained by the Detrended cross correlation coefficient method showed that for both returns and volatility the correlations between the series are positive, increase with time scale and are stronger for the series of returns.
\end{abstract}

Keywords: Mango Tommy, Palmer; Correlations; Detrended fluctuation analysis; Detrended cross correlation coefficient.

\section{Resumen}

En las últimas décadas, el comercio de frutas en Brasil ha crecido significativamente, atendiendo al mercado interno y externo. Entre las principales frutas producidas y comercializadas, destaca el mango, la fruta más exportada en Brasil. 
En este trabajo se analizó la serie temporal de retorno y la volatilidad semanal de precios de dos variedades de mango, Palmer y Tommy Atkins, producidas en el Valle de São Francisco, la región con mayor producción de mango del país. Para calcular los exponentes de escala de las autocorrelaciones y las correlaciones cruzadas entre las series analizadas se utilizaron los métodos Detrended Fluctuation Analysis (DFA) y Detrended Cross Correlation Analysis (DCCA). Los resultados mostraron que la serie de volatilidad tiene una persistencia más fuerte que la serie de retorno que presentó dos regímenes de invarianza de escala con correlaciones anti-persistentes en las escalas mayores. Las correlaciones cruzadas entre las series de retorno también muestran dos regímenes de escala con exponentes similares a la serie de retorno de la variedad Tommy. Los valores del coeficiente de correlación obtenidos por el método Detrended Cross Correlation Coefficient mostraron que tanto para los retornos como para la volatilidad las correlaciones entre las series son positivas, aumentan con la escala de tiempo y son más fuertes para la serie de retorno.

Palabras clave: Mango Tommy, Palmer; Correlaciones; Detrended fluctuation analysis; Detrended cross correlation coefficient.

\section{Introdução}

O comércio de frutas cresceu expressivamente nas últimas décadas e a fruticultura nacional participa diretamente na economia atendendo ao mercado interno e externo. Gerando milhões de empregos diretos, o setor fruticultor se destaca dentro do agro sendo responsável por $16 \%$ da mão de obra. No Brasil a produção total de frutas in natura foi superior a 44 milhões de toneladas em 2020, mantendo o posto de terceiro maior produtor mundial de frutas, ficando atrás apenas da China e Índia, com produções estimadas em 265 e 93 milhões de toneladas em 2018, respectivamente (Anuário Brasileiro de Horti\&Fruti, 2021).

Dentre as principais frutas produzidas e comercializadas no país, destaca-se a manga. Originária do Sudeste Asiático, a cultura da manga se disseminou para várias regiões do mundo, encontrando no Brasil excelentes condições para seu desenvolvimento e produção (Souza et al., 2002). A manga é cultivada nas cinco regiões brasileiras e mediante os avanços tecnológicos é produzida durante todo o ano, com um grande destaque para a produção na região nordeste, que a partir da década de 2000 passou a ser responsável por cerca de 2/3 de toda a produção nacional (Valadares \& Landau, 2020).

Segundo o Anuário Brasileiro de Horti\&Fruti (2021), a manga é a fruta mais exportada pelo Brasil, alcançando o valor de 246,9 milhões de dólares em mais de 243 milhões de quilogramas exportados em 2020, o que corrobora sua influência no mercado agrícola. A principal responsável por esses números expressivos de exportação da manga nacional é a região do Vale do São Francisco, situada em pleno Semiárido Nordestino, exportando 212,2 mil toneladas no último ano, correspondendo a 87\% do total exportado da fruta, segundo dados do Observatório do Mercado de Manga da Embrapa Semiárido (PE), a partir de dados do Comex Stat (MDIC).

É relevante ressaltar que as commodities agrícolas são vulneráveis a instabilidades provocadas por flutuações sazonais dos preços, sendo assim, é necessário conhecer o padrão dessas flutuações sazonais e a volatilidade dos preços, bem como os principais fatores influenciadores. Segundo Menezes (2015) os principais fatores influenciadores dos preços das commodities são (oferta): custos de produção, fatores climáticos, políticas de incentivos à produção de biocombustíveis, informações divulgadas por agências internacionais e níveis de estoque mundial; (demanda): crescimento populacional e desenvolvimento econômico de países emergentes, variações das taxas cambiais, derivativos - contratos futuros, efeitos especulativos de mercados financeiros, níveis de estoque mundial e preços observados em outras bolsas internacionais.

Das exportações de manga provenientes do Vale do São Francisco, grande parte é destinada aos mercados europeu e americano, onde os preços dos mercados interno e americano sofrem influência dos preços do mercado europeu, fazendo com que esse tenha comportamento de líder na cadeia, e as variações nos seus preços sejam transmitidas aos demais (Souza et al., 2018).

Diante de tantos empecilhos para o estudo da relação entre preços dos mercados agrícolas, torna-se difícil a escolha de uma abordagem quantitativa apropriada. Comumente são utilizados métodos estatísticos para estudar correlações e transmissões de preços entre mercados agrícolas (Beckmann \& Czudaj, 2014; Nicola, De Pace, \& Hernandez, 2016), porém métodos e modelos baseados na mecânica estatística, no campo da Econofísica, nos trazem novos conhecimentos e nos ajudam a melhorar 
a compreensão das variações e relações dos preços das commodities agrícolas (Liu, 2014; Pal \& Mitra, 2018; Lima et al., 2019).

Tendo em vista esses relatos, o estudo aqui realizado tem como objetivo contribuir para melhor entendimento da evolução temporal dos preços de mangas produzidas no Vale do São Francisco ao longo das últimas duas décadas, das correlações de longo alcance e de correlações cruzadas entre seus preços. Para isso serão empregadas as técnicas da Econofísica para séries não-estacionárias, Detrended Fluctuation Analysis (DFA) (Peng et al., 1994), Detrended Cross Correlation Analysis (DCCA) (Podobnik \& Stanley, 2008), e o Detrended Cross Correlation Coefficient $\left(\rho_{\mathrm{DCCA}}\right)$ (Zebende, 2011). Em particular, serão analisadas as séries semanais de preços de duas variedades: Tommy Atkins, que ganha espaço na exportação por possuir características essenciais relacionadas a fatores pós-colheita, como resistência ao manuseio e transporte e a variedade Palmer, por apresentar aumento significativo de sua oferta (de Oliveira Araújo, Moraes, \& de Carvalho, 2017).

\section{Metodologia}

O presente estudo é uma pesquisa de natureza quantitativa (Pereira et al., 2018), investigam-se as possíveis correlações de longo prazo entre as séries temporais dos preços de duas variedades de manga produzidas no Vale do São Francisco. Foram aplicados os métodos DFA e DCCA para obtenção da variância sem tendência (DFA) e a covariância sem tendência (DCCA) que foram utilizadas para obtenção do coeficiente de correlação cruzada sem tendência $\left(\rho_{D C C A}\right)$. Para implementação desses métodos, foram usados um software próprio, desenvolvido nas linguagens $\mathrm{C}$ de programação, e o software $\mathrm{R}$ (versão 4.1.0).

\subsection{Descrição dos dados}

Os dados utilizados foram os preços semanais expressos em reais por quilograma $(\mathrm{R} \$ / \mathrm{kg})$ das variedades de manga Tommy Atkins e Palmer, produzidas na região do Vale do São Francisco, registrados no período de 21 de março de 2001 a 18 de junho de 2021. As séries de dados foram obtidas no Centro de Estudos Avançados em Economia Aplicada / Escola Superior de Agricultura Luiz de Queiroz / Universidade de São Paulo - CEPEA / ESALQ / USP (CEPEA/ESALQ/USP, 2021). Foram analisadas as séries temporais de retornos $\mathrm{R}_{\mathrm{t}}=\ln \left(\mathrm{P}_{\mathrm{t}}\right)-\ln \left(\mathrm{P}_{\mathrm{t}-1}\right)$ e de volatilidade $\mathrm{V}_{\mathrm{t}}=\left|\mathrm{R}_{\mathrm{t}}\right|$ em que $P_{t}$ é o preco de uma commodity na semana t. As séries temporais originais, de retornos e da volatilidade dos preços de manga Tommy Atkins e Palmer são apresentadas na Figura 1, Figura 2 e Figura 3, respectivamente.

A Figura 1 apresenta o comportamento das séries temporais originais dos preços semanais das variedades de manga Tommy Atkins e Palmer para o intervalo explorado. Em ambas variedades se nota uma concentração dos preços, entre $\mathrm{R} \$ 0,50$ e R \$ 2,50 para a Palmer e entre R\$ 0,20 e R\$ 1,50 no caso da Tommy Atkins, durante a década 2005-2015, havendo aumento após esse intervalo alcançando valores superiores a $\mathrm{R} \$ 4,00$ no início do primeiro semestre de 2016. Esse aumento significativo é resultado de um período de baixa oferta, escassez da fruta no mercado nacional, uma vez que o clima não estava favorável às floradas. Observa-se uma diminuição nas cotações médias de ambas variedades em volta do final do primeiro semestre de 2016, aproximando-se da normalidade histórica de preços para a região, isso se deu pela elevação da oferta nacional e diminuição da demanda nas regiões Sul e Sudeste do País. Apresentam tendência de alta de preços ao final do ano 2019 e início de 2020, sendo as exportações o foco dos produtores da região, pelo fato do câmbio R \$ - US\$ bastante favorável, atingindo novos recordes de exportações. 
Figura 1. Séries temporais originais dos preços das mangas Palmer e Tommy.
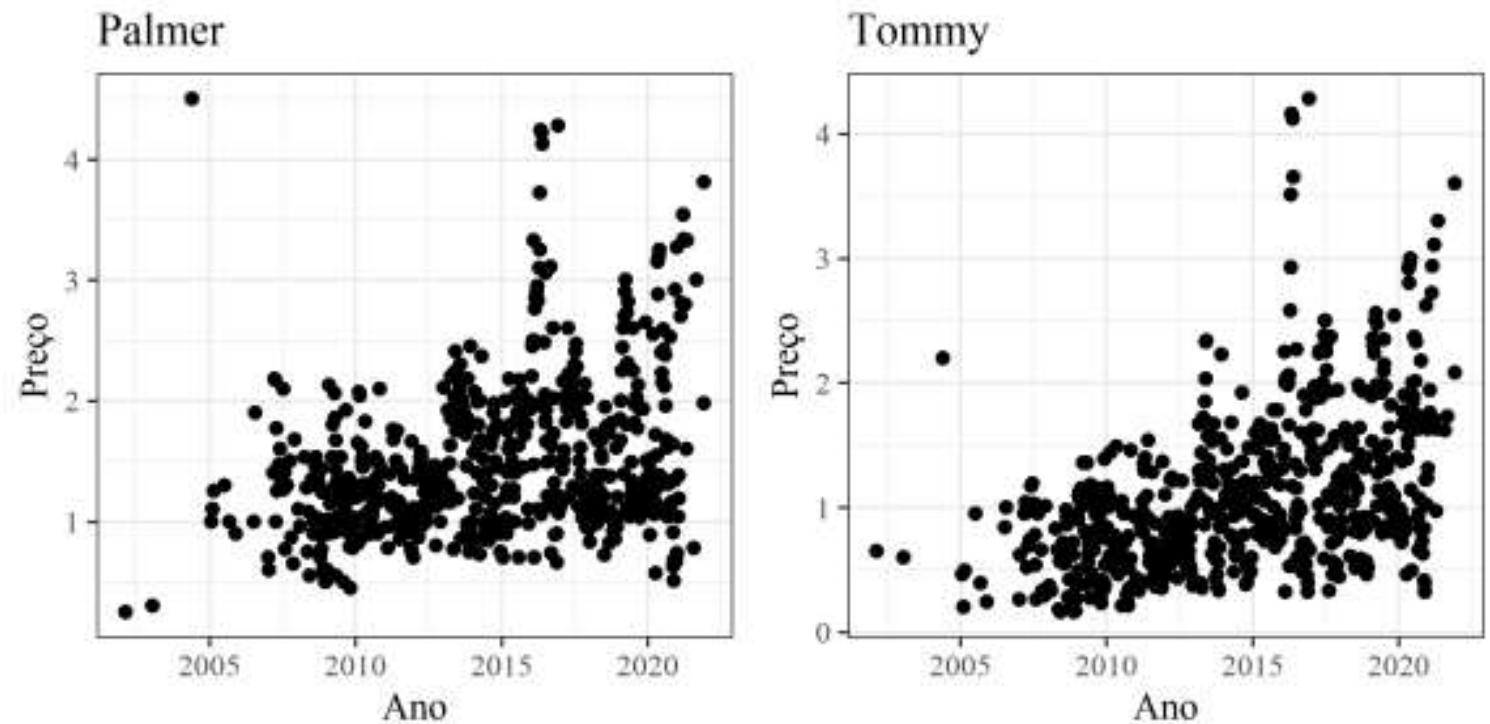

Fonte: Autores, a partir dos dados do CEPEA.

A Figura 2 apresenta o comportamento das séries temporais de retornos dos preços semanais das variedades de manga Tommy Atkins e Palmer para o intervalo explorado. Observa-se que há maiores valores de retorno anteriormente a 2010 para a variedade Palmer, após esse período segue-se uma tendência em volta dos valores -0,2 e 0,2. Diferentemente da variedade citada, a Tommy apresenta valores mais discrepantes mesmo após 2010.

Figura 2. Séries temporais de retornos dos preços das mangas Palmer e Tommy.
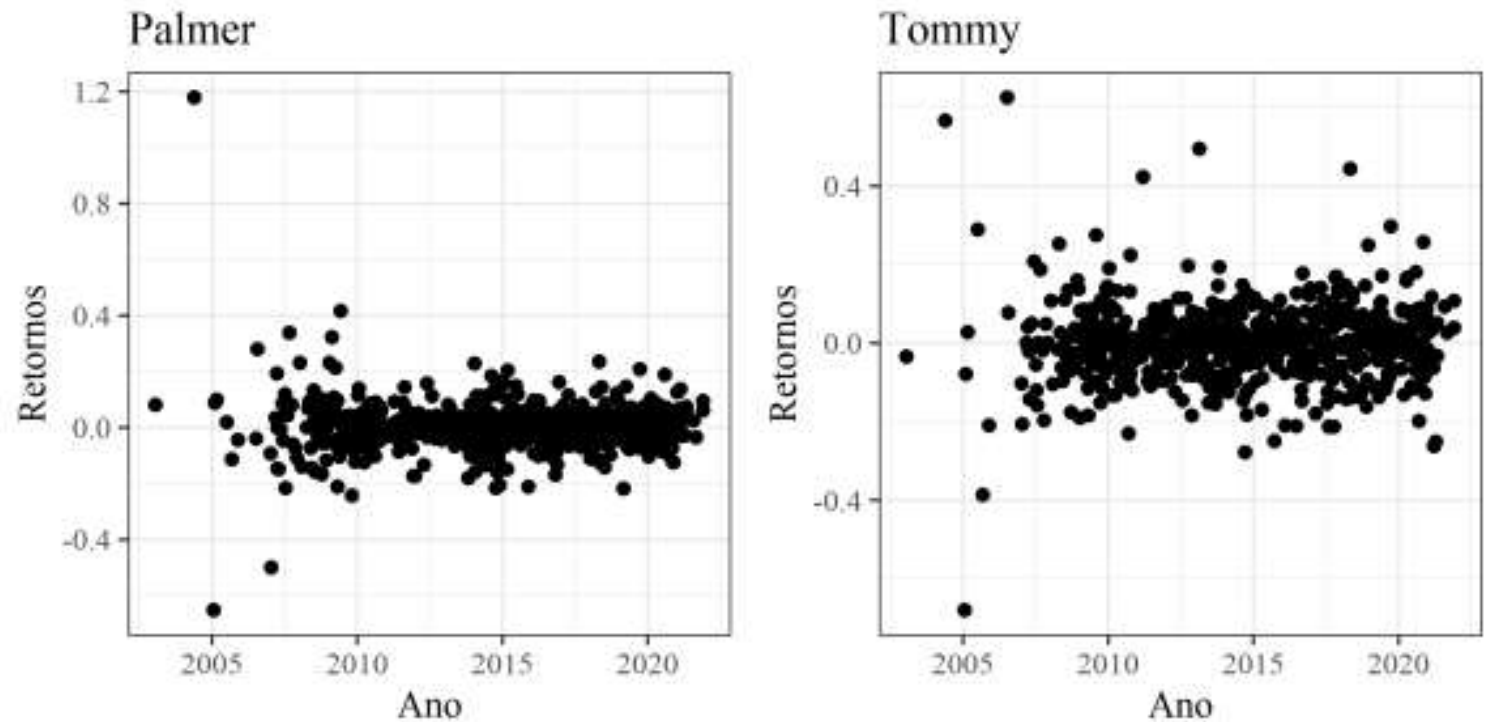

Fonte: Autores, a partir dos dados do CEPEA.

A Figura 3 apresenta o comportamento das séries temporais de volatilidade dos preços semanais das variedades de manga Tommy Atkins e Palmer para o intervalo explorado. Nota-se que a volatilidade é maior para ambas as variedades em torno de 2005, sendo mantida a tendência de baixa volatilidade a partir de 2010 para a variedade Palmer. A variedade Tommy 
apresenta picos de volatilidade mesmo após 2010, chegando a superar o valor de 0,4.

Figura 3. Séries temporais de volatilidade dos preços das mangas Palmer e Tommy.

Palmer

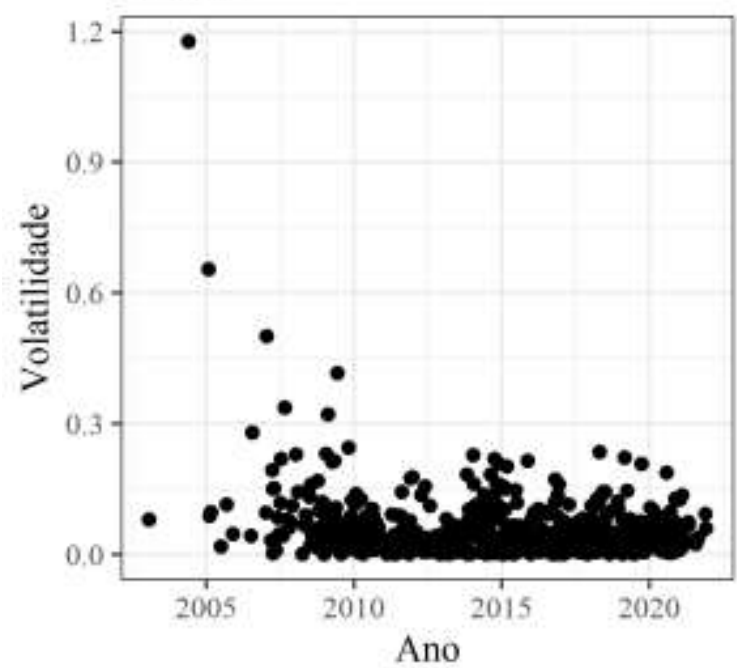

Tommy

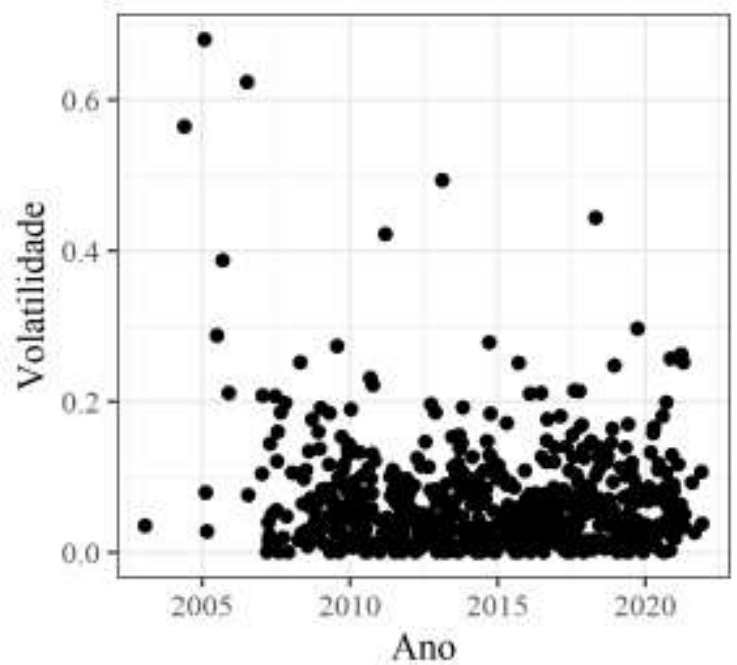

Fonte: Autores, a partir dos dados do CEPEA.

\subsection{Detrended Fluctuation Analysis (DFA)}

Introduzida por Peng et al. (1994) em um estudo de correlações de longo alcance em sequências de DNA, a análise de flutuação sem tendência (Detrended Fluctuation Analysis - DFA) consolidou-se como um método poderoso para a quantificação de correlações de longo alcance em uma série temporal que não apresente estacionariedade. Esse método foi aplicado com sucesso em diversos estudos de variadas áreas, por exemplo: Climatologia (Matsoukas, Islam, \& Rodriguez-Iturbe, 2000; Thaxton et al., 2018), Medicina (Willson et al., 2002; Jospin et al., 2007), Geofísica (Zheng et al., 2012), Ecologia (Stosic et al., 2016) e Finanças (Lahmiri, 2015).

A implementação do algoritmo DFA pode ser descrita a partir de cinco passos:

1 - Considere a série temporal $\left\{x_{i}\right\}$ com $\mathrm{i}=1, \ldots, \mathrm{N}$ (comprimento). Integra-se $x_{i}$ para obter

$$
X_{k}=\sum_{i=1}^{k}\left[x_{i}-\langle x\rangle\right],
$$

sendo $\langle x\rangle$ o valor médio da série, ou seja, $\langle x\rangle=\frac{1}{\mathrm{~N}} \sum_{i=1}^{N} x_{i}$, com $\mathrm{k}=1, \ldots, \mathrm{N}$;

2 - A série integrada $X_{k}$ é dividida em caixas de igual comprimento n (escala temporal). Neste estudo utilizamos caixas sobrepostas com início em $N-n+1$ dados sucessivos;

3 - Para cada caixa $v=1, \ldots, N-n+1$ calcula-se a tendência local (ajuste linear) $X_{k, v}$ e é subtraída da série integrada $X_{k}$, obtendo-se a variância sem tendência

$$
f_{D F A}^{2}(n, v)=\frac{1}{n} \sum_{k=v}^{v+n-1}\left(X_{k}-X_{k, v}\right)^{2} ;
$$

4 - Depois, para uma dada escala temporal de comprimento $\mathrm{n}$ a função de flutuação, $F_{D F A}(n)$, é calculada por: 


$$
F_{D F A}(n)=\sqrt{\frac{1}{(N-n+1)} \sum_{v=1}^{N-n+1} f_{D F A}^{2}(n, v)} ;
$$

5 - Os passos acima são repetidos para uma gama de escalas temporais compreendidas por, $10 \leq n \leq \frac{N}{4}$, para finalmente obterse uma relação entre $F_{D F A} \times n$.

Havendo correlações de longo prazo na série original, $F_{D F A}(n)$ aumentará com $n$ de acordo com uma lei de potência $F_{D F A}(n) \sim n^{\alpha}$ e o expoente escalar $\alpha$ é obtido como a inclinação da regressão linear de $\log F_{D F A}(n)$ versus $\log n$. Sendo $0<$ $\alpha<1$, o expoente escalar $\alpha$ é igual ao expoente de Hurst $H$ descrevendo autocorrelações nas séries originais. São três possibilidades indicadas a partir desse expoente escalar:

- Ausência de correlações: $\mathrm{O}$ valor $\boldsymbol{\alpha}=\mathbf{0 , 5}$ indica a ausência de correlações (ruído branco);

- Correlações persistentes: $\mathrm{O}$ valor $\boldsymbol{\alpha}>\mathbf{0 , 5}$ indica correlações persistentes a longo prazo, ou seja, valores grandes (pequenos) têm maior probabilidade de serem seguidos por valores grandes (pequenos);

- Correlações antipersistentes: $\mathrm{O}$ valor $\boldsymbol{\alpha}<\mathbf{0 , 5}$ indica correlações antipersistentes a longo prazo, ou seja, valores pequenos têm maior probabilidade de serem seguidos por valores grandes e vice-versa.

Caso $1<\alpha<2$, o expoente escalar $\alpha$ indica movimento browniano fracionário com incrementos descritos pelo expoente de Hurst $H=\alpha-1$, sendo os valores $\alpha=1$ e $\alpha=1,5$ correspondentes ao ruído $\frac{1}{f}$ e ao ruído browniano (integração do ruído branco), respectivamente (Siqueira et al., 2010).

\subsection{Detrended Cross Correlation Analysis (DCCA)}

Introduzida por Podobnik e Stanley (Podobnik \& Stanley, 2008) como uma generalização do método DFA, a análise de correlação cruzada sem tendência (Detrended Cross-Correlation Analysis - DCCA) é útil para mensurar correlações entre duas séries temporais simultâneas. Assim como o DFA, esse método foi amplamente aplicado em diversas áreas como Geofísica (Marinho, Sousa, \& Andrade, 2013), Fisiologia (Zhao et al., 2013), Climatologia (Vassoler \& Zebende, 2012), Hidrologia (Dey \& Mujumdar, 2018) e Finanças (Shin, Lim, \& Min, 2020).

A implementação do algoritmo DCCA pode ser descrita a partir de cinco passos:

1 - Considere duas séries temporais $\left\{x_{i}\right\}$ e $\left\{y_{i}\right\}$ com $i=1, \ldots, N$ (comprimento). Integra-se $x_{i}$ e $y_{i}$ para obter

$$
X_{k}=\sum_{i=1}^{k}\left[x_{i}-\langle x\rangle\right] \text { e } Y_{k}=\sum_{i=1}^{k}\left[y_{i}-\langle y\rangle\right],
$$

sendo $\langle x\rangle$ e $\langle y\rangle$ os valores médios das séries $\left\{x_{i}\right\}$ e $\left\{y_{i}\right\}$, ou seja, $\langle x\rangle=\frac{1}{\mathrm{~N}} \sum_{i=1}^{N} x_{i}$ e $\langle y\rangle=\frac{1}{\mathrm{~N}} \sum_{i=1}^{N} y_{i}$, com k $=1, \ldots, \mathrm{N}$;

2 - As séries integradas $X_{k}$ e $Y_{k}$ são divididas em $(N-n+1)$ caixas (sobrepostas) de igual comprimento $\mathrm{n}$ (escala temporal); 3 - Para cada caixa $v=1, \ldots, N-n+1$ calculam-se as tendências locais (ajuste linear) em $X_{k}$ e $Y_{k}$, obtendo $X_{k, v}$ e $Y_{k, v}$ e são subtraídas das séries integradas $X_{k}$ e $Y_{k}$, respectivamente, obtendo-se

$$
f_{D C C A}^{2}(n, v)=\frac{1}{n} \sum_{k=v}^{v+n-1}\left(X_{k}-X_{k, v}\right)\left(Y_{k}-Y_{k, v}\right) ;
$$

4 - Depois, para uma dada escala temporal de comprimento n a covariância sem tendência $F_{D C C A}^{2}(n)$, é calculada por:

$$
\mathrm{F}_{\mathrm{DCCA}}^{2}(\mathrm{n})=\frac{1}{(\mathrm{~N}-\mathrm{n}+1)} \sum_{v=1}^{N-n+1} f_{D C C A}^{2}(n, v) ;
$$


5 - Os passos acima são repetidos para uma gama de escalas temporais compreendidas por, $10 \leq n \leq \frac{N}{4}$, para finalmente obterse uma relação entre $F_{D C C A} \times n$.

Havendo correlações de longo prazo nas séries originais, $F_{D C C A}(n)$ aumentará com $n$ de acordo com uma lei de potência $F_{D C C A}(n) \sim n^{\lambda}$. Com a inclinação da regressão linear de $\log F_{D C C A}(n)$ versus $\log n$ é possível obter o expoente escalar $\lambda$, cuja interpretação é similar à do expoente $\alpha$ do método DFA. Se existir em cada série uma longa memória dos seus valores anteriores, tal como uma longa memória de valores anteriores da outra série, isso implicará na presença de correlações cruzadas de longo prazo entre duas séries simultâneas.

\subsection{Detrended Cross Correlation Coefficient $\left(\rho_{D C C A}\right)$}

Introduzido por Gillney Figueira Zebende (Zebende, 2011) o coeficiente de correlação cruzada (Detrended Cross Correlation Coefficient $-\rho_{D C C A}$ ) é expresso da seguinte forma

$$
\rho_{D C C A}(n)=\frac{F_{D C C A}^{2}(n)}{F_{D F A_{1}}(n) F_{D F A_{2}}(n)},
$$

em que $F_{D C C A}^{2}(n)$ é a covariância sem tendência obtida pelo método DCCA, enquanto $F_{D F A_{1}}(n)$ e $F_{D F A_{2}}(n)$ retratam as funções de flutuação obtidas pelo método DFA. Foi utilizado em séries climáticas (Vassolar \& Zebende, 2012), na fisiologia (Chen et al., 2018) e dados financeiros (Pessoa et al., 2021). O coeficiente pode variar entre o valor mínimo -1 (correlação cruzada negativa perfeita) e o valor máximo 1 (correlação cruzada positiva perfeita), análogo ao coeficiente de Pearson.

Caso as séries analisadas não apresentem correlação cruzada, $\rho_{D C C A}(n)$ oscila próximo a zero, em contrapartida para séries anticorrelacionadas, $\rho_{D C C A}(n)$ é estritamente negativo e para séries correlacionadas positivamente, $\rho_{D C C A}(n)$ é positivo. Havendo correlações de longo alcance entre as séries examinadas, o coeficiente segue a lei de potência $\rho_{D C C A}(n) \sim \mathrm{n}^{\omega}$, sendo $\omega=2 \lambda-\alpha_{1}-\alpha_{2}$ (Zebende et al., 2013).

\section{Resultados e Discussão}

Pretendendo alcançar o objetivo desse estudo, a seguir serão apresentados os resultados provenientes das observações das séries temporais de retorno e de volatilidade dos preços das variedades de manga Tommy Atkins e Palmer, produzidas no Vale do São Francisco. As análises dos expoentes $(\alpha)$ e ( $\lambda)$ podem ser vistas nas Figuras 4 e 5 para séries de retorno e volatilidade dos preços, respectivamente.

Na Figura 4 vemos que os retornos de preços de ambas variedades apresentam ausência das correlações de longo alcance $(\alpha \sim 0,5)$ até a escala temporal de 66 semanas $\left(\log _{10}(s) \sim 1,75\right)$ para a variedade Palmer e até a escala de 70 semanas $\left(\log _{10}(s) \sim 1,84\right)$ para a variedade Tommy. A partir destas escalas as séries de retornos de preços apresentam correlações de longo alcance antipersistentes $(\alpha<0,5)$, ou seja, uma tendência de crescimento implicará numa tendência de redução e vice versa. As correlações cruzadas (figura $4 \mathrm{C})$ apresentam persistência $(\lambda>0,5)$ até a escala de 70 semanas $\left(\log _{10}(s) \sim 1,84\right)$, seguindo $\operatorname{com} \lambda<0,5$ indicando que nas escalas maiores de 70 semanas as correlações cruzadas são antipersistentes $-\mathrm{o}$ aumento (diminuição) dos preços de uma variedade tem maior probabilidade de ser seguida com diminuição (aumento) de preços de ambas variedades. A escala temporal que divide os dois regimes de escala (efeito crossover) e os valores de expoentes da escala $\lambda$ são mais próximos aos expoentes da escala $\alpha$ da variedade Tommy, indicando que as flutuações de preços desta tem maior influência nas flutuações de preços da variedade Palmer. Este resultado está de acordo com os obtidos por Lima et al. (2013), onde encontraram que os preços de manga são diretamente influenciados por variáveis externas, como a taxa de câmbio e os preços externos. A variedade Tommy Atkins que responde bem às técnicas de manejo, suporta o tratamento pós-colheita de transporte e é atrativa visualmente, tem grande espaço no mercado da União Europeia e no mercado norte-americano (de Oliveira 
Araújo, Moraes, \& de Carvalho, 2017). Por isso os preços desta variedade flutuam de acordo com mercado externo que afeta diretamente a oferta no mercado interno e os preços de outras variedades desta fruta.

Figura 4. Análise DFA e DCCA das séries de retornos das mangas Palmer e Tommy.
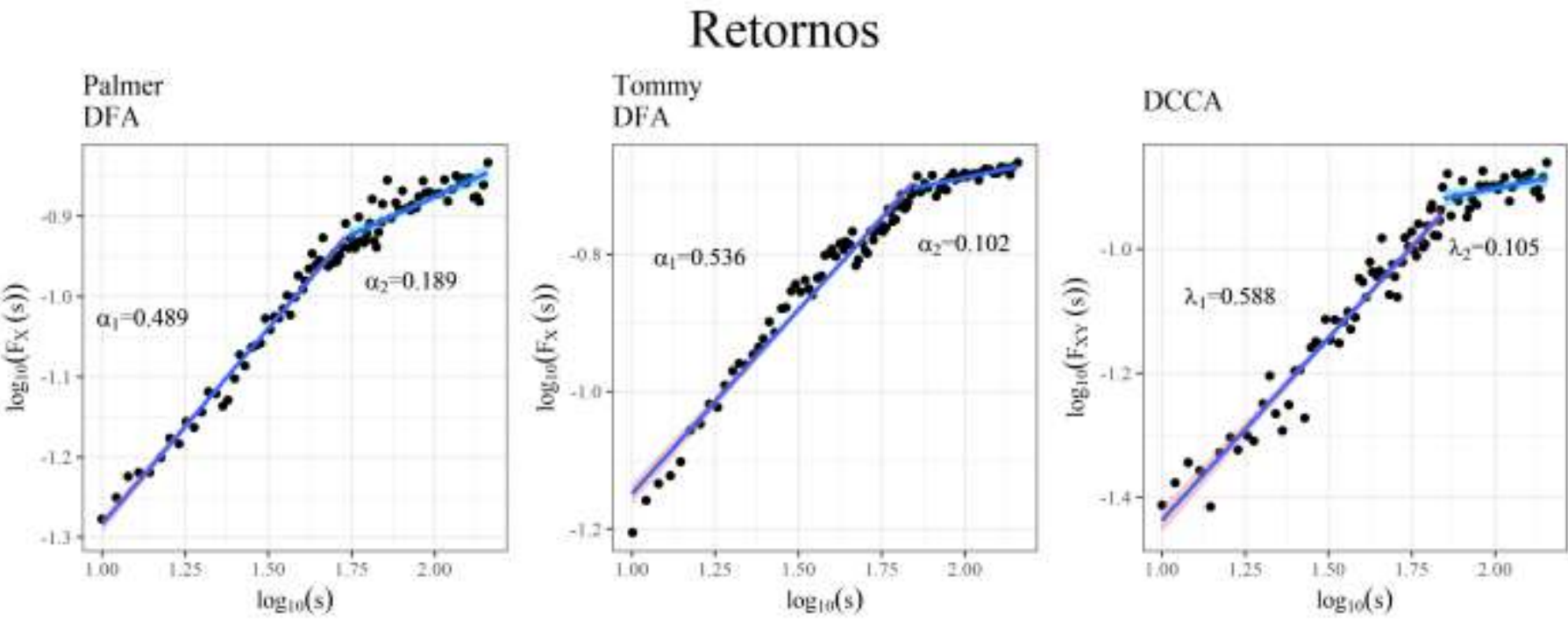

Fonte: Autores, a partir dos dados do CEPEA.

Na Figura 5 pode-se observar que os expoentes da escala DFA $(\alpha)$ e DCCA $(\lambda)$, que são resultantes do cálculo do coeficiente angular das respectivas retas dos gráficos, são maiores que 0,5 , ou seja, indicam a presença de correlações de longo alcance persistente em séries de volatilidade das variedades de manga Palmer e Tommy. A persistência é mais forte para a variedade Tommy (indicado por maior valor de expoente) que sofre mais interferência de fatores externos. Também é possível notar a presença de correlação cruzada de longo alcance persistente entre essas duas variedades, evidenciando a ligação existente entre essas commodities agrícolas.

Figura 5. Análise DFA e DCCA das séries de volatilidade das mangas Palmer e Tommy.

\section{Volatilidade}
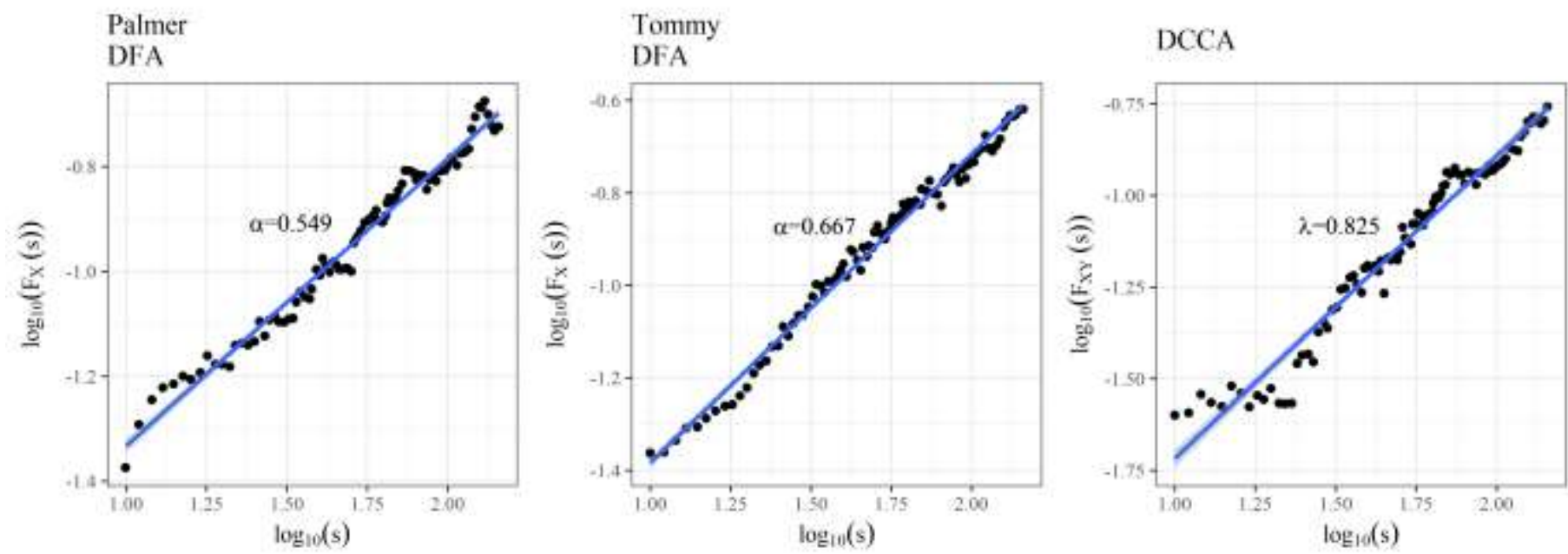

Fonte: Autores, a partir dos dados do CEPEA. 
Nas Figuras 6 e 7 são apresentados os coeficientes de correlação DCCA $\left(\rho_{D C C A}\right)$ entre as séries de retornos e volatilidade dos preços, respectivamente.

Na Figura 6 nota-se que as séries de retornos são correlacionadas positivamente $\left(\rho_{D C C A}>0\right)$ durante todo o período analisado, havendo uma diminuição na correlação para escalas menores, mas cresce na medida em que a escala temporal aumenta. A partir da escala de 20 semanas $\left(\log _{10}(s) \sim 1,3\right)$ há um crescimento mais acentuado que se prolonga até a escala de 87 semanas $\left(\log _{10}(s) \sim 1,94\right)$, onde se mantém com $\rho_{D C C A}>0,55$.

Figura 6. Coeficientes DCCA e das séries de retornos das mangas Palmer e Tommy.

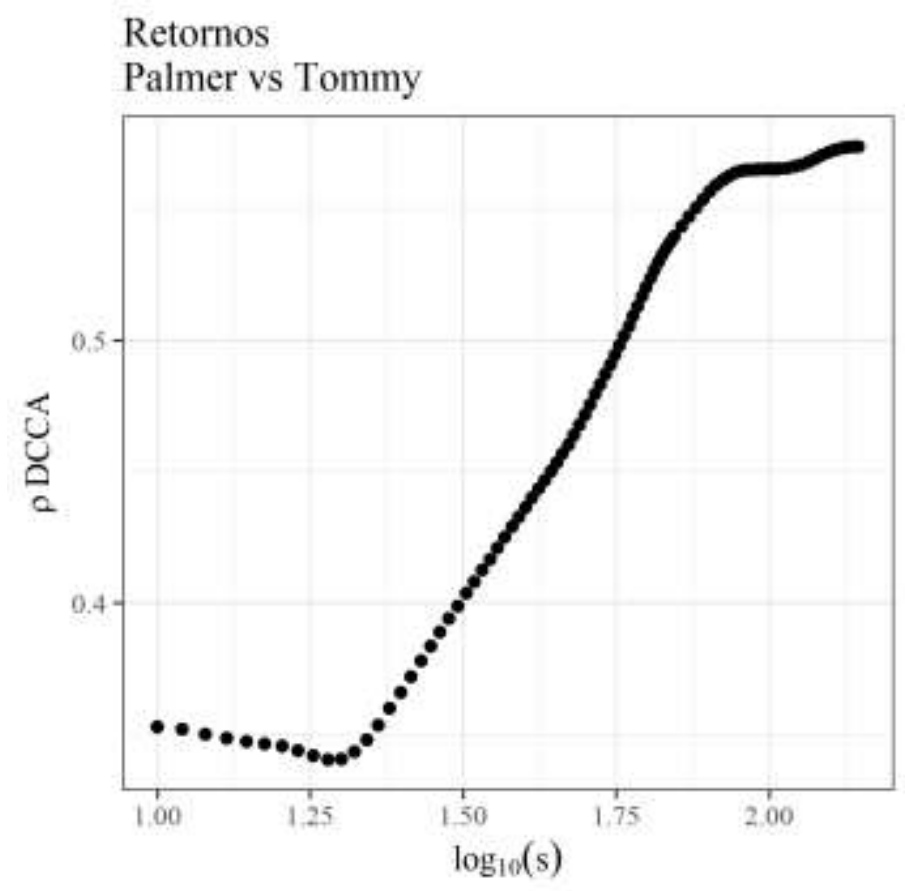

Fonte: Autores, a partir dos dados do CEPEA.

Na Figura 7 é apresentado o coeficiente DCCA $\left(\rho_{D C C A}\right)$ entre as séries de volatilidade dos preços das variedades de manga Palmer e Tommy Atkins. O coeficiente de correlação cruzada de volatilidade se comportou de forma similar ao de retorno dos preços, havendo uma diminuição para escalas menores e depois um notório crescimento, porém não chegou a atingir o mesmo patamar do coeficiente observado anteriormente, sendo inferior desde o início do período analisado, evidenciando que as séries de retorno dos preços possuem correlações mais fortes. Observa-se que há uma diminuição a partir da escala de 115 semanas $\left(\log _{10}(s) \sim 2,06\right)$, mas mantendo-se em $\rho_{D C C A}>0,37$. 
Figura 7. Coeficientes DCCA das séries de volatilidade das mangas Palmer e Tommy.

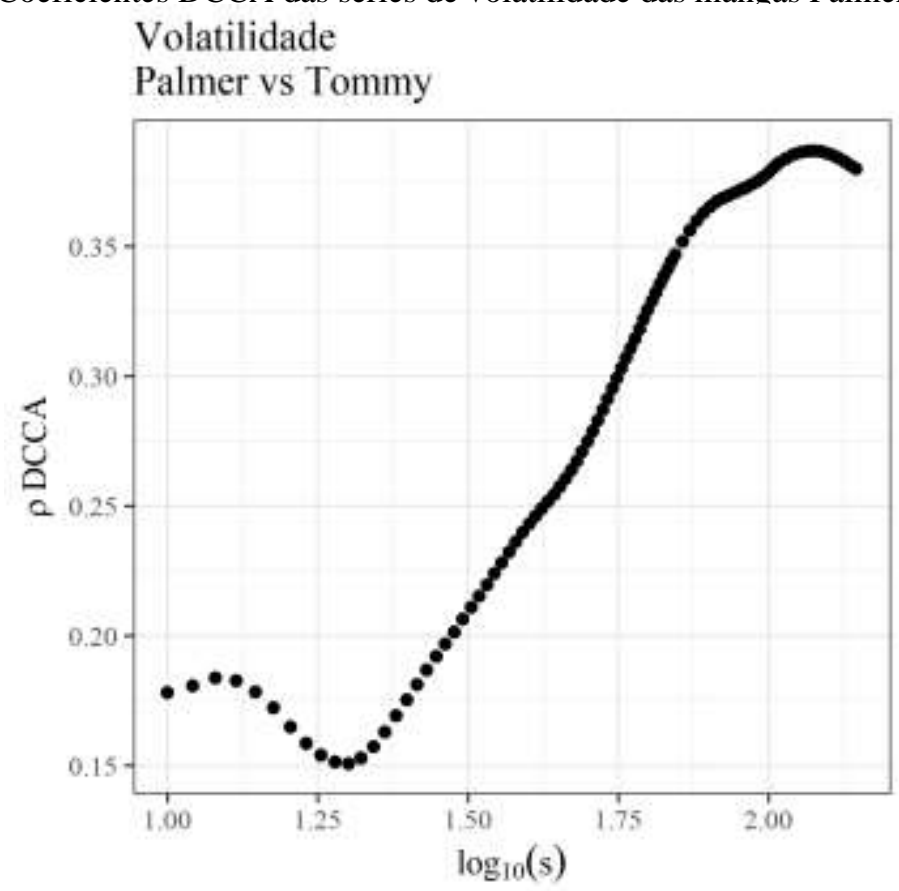

Fonte: Autores, a partir dos dados do CEPEA.

\section{Considerações Finais}

No decorrer do trabalho foram analisadas as séries temporais originais, de retorno e de volatilidade de preços das variedades de manga: Palmer e Tommy Atkins. Foram realizadas análises das correlações e correlações cruzadas entre séries temporais de retorno e de volatilidade com uso dos métodos DFA e DCCA, sendo os resultados obtidos usados para o cálculo do coeficiente de correlação $\rho_{D C C A}$, útil para medir os níveis dessas correlações.

Os resultados apresentaram maior persistência para as séries de volatilidade, dois regimes para as séries de retorno, com antipersistência para maiores escalas. Correlações cruzadas entre as séries de retornos também apresentam dois regimes de escala com expoentes semelhantes as séries de retornos da variedade Tommy, indicando que as flutuações de preços desta variedade, que lidera na exportação de manga, afetam mais os preços no mercado interno (variedade Palmer) do que o contrário. Os valores do coeficiente de correlação apontaram para uma correlação mais forte entre as séries de retornos, apesar de um comportamento semelhante para a correlação das séries de volatilidade dos preços.

Os resultados deste trabalho contribuem para um melhor entendimento do comportamento das flutuações de preços de manga, podendo ser úteis para produtores e exportadores de manga em suas tomadas de decisões.

Para futuros trabalhos, é viável uma análise mais profunda dessa e de outras frutas produzidas no Vale do São Francisco e em outros polos fruticultores, aprofundando-se na análise de correlações e utilizando outras técnicas como Multifractal Detrended Cross-Correlation Analysis (MF-DCCA).

\section{Agradecimentos}

Os autores agradecem o suporte e encorajamento oferecidos pela Universidade Federal Rural de Pernambuco (UFRPE), pelo Programa de Pós-Graduação em Biometria e Estatística Aplicada (PPGBEA), pela Coordenação de Aperfeiçoamento de Pessoal de Nível Superior (CAPES), pelo Conselho Nacional de Desenvolvimento Científico e Tecnológico (CNPq) e pela Fundação de Amparo à Ciência e Tecnologia do Estado de Pernambuco (FACEPE). Agradecemos pela 
disponibilidade dos dados ao Centro de Estudos Avançados em Economia Aplicada (CEPEA).

\section{Referências}

Anuário brasileiro de horti\&fruti 2021 (2021). BrazilianHorti\&FrutiYearbook. Editora Gazeta Santa Cruz. 104p.

Beckmann, J., \& Czudaj, R. (2014). Volatility transmission in agricultural futures markets. Economic Modelling, 36, 541-546.

CEPEA/ESALQ/USP. (2020). Consultas ao Banco de Dados do Site - Centro de Estudos Avançados em Economia Aplicada - CEPEA-Esalq/USP. https://www.cepea.esalq.usp.br/br/consultas-ao-banco-de-dados-do-site.aspx

Chen, Y., Cai, L., Wang, R., Song, Z., Deng, B., Wang, J., \& Yu, H. (2018). DCCA cross-correlation coefficients reveals the change of both synchronization and oscillation in EEG of Alzheimer disease patients. Physica A: Statistical Mechanics and its Applications, 490, 171-184.

de Nicola, F., De Pace, P., \& Hernandez, M. A. (2016). Co-movement of major energy, agricultural, and food commodity price returns: A time-series assessment. Energy Economics, 57, 28-41.

de Oliveira Araújo, D., Moraes, J. A. A., \& de Carvalho, J. L. M. (2017). Fatores determinantes na mudança do padrão de produção e consumo da manga no mercado nacional. Revista Em Agronegocio e Meio Ambiente, 10, 51-73.

Dey, P., \& Mujumdar, P. P. (2018). Multiscale evolution of persistence of rainfall and streamflow. Advances in water resources, 121, 285-303.

Jospin, M., Caminal, P., Jensen, E. W., Litvan, H., Vallverdú, M., Struys, M. M., \& Kaplan, D. T. (2007). Detrended fluctuation analysis of EEG as a measure of depth of anesthesia. IEEE transactions on biomedical engineering, 54(5), 840-846.

Lahmiri, S. (2015). Long memory in international financial markets trends and short movements during 2008 financial crisis based on variational mode decomposition and detrended fluctuation analysis. Physica A: Statistical Mechanics and its Applications, 437, 130-138.

Lima, C. R. A., de Melo, G. R., Stosic, B., \& Stosic, T. (2019). Cross-correlations between Brazilian biofuel and food market: Ethanol versus sugar. Physica A: Statistical Mechanics and its Applications, 513, 687-693.

Lima, J. R., de Sales Silva, J., \& Santos, R. K. B. (2013). Comportamento dos preços da manga exportada do Brasil: 2004-2012. Organizações Rurais \& Agroindustriais, 15(3).

Liu, L. (2014). Cross-correlations between crude oil and agricultural commodity markets. Physica A: Statistical Mechanics and its Applications, 395, $293-302$.

Marinho, E. B. S., Sousa, A. M. Y. R., \& Andrade, R. F. S. (2013). Using detrended cross-correlation analysis in geophysical data. Physica A: Statistical Mechanics and its Applications, 392(9), 2195-2201.

Matsoukas, C., Islam, S., \& Rodriguez-Iturbe, I. (2000). Detrended fluctuation analysis of rainfall and streamflow time series. Journal of Geophysical Research: Atmospheres, 105(D23), 29165-29172.

Menezes, I. D. (2015). Revisão da literatura empírica acerca das variáveis que impactam a precificação de commodities agrícolas: soja, milho, café e boi gordo.

Pal, D., \& Mitra, S. K. (2018). Interdependence between crude oil and world food prices: A detrended cross correlation analysis. Physica A: Statistical Mechanics and its Applications, 492, 1032-1044.

Peng, C. K., Buldyrev, S. V., Havlin, S., Simons, M., Stanley, H. E., \& Goldberger, A. L. (1994). Mosaic organization of DNA nucleotides. Physical review $e, 49(2), 1685$.

Pereira, A. S., Shitsuka, D. M., Parreira, F. J., \& Shitsuka, R. (2018). Metodologia da pesquisa científica. UFSM. https://repositorio. ufsm. br/bitstream/handle/1/15824/Lic_Computacao_Metodologia-Pesquisa-Cientifica. pdf.

Pessoa, R. V. S., de Carvalho Barreto, I. D., da Silva Araújo, L., Moreira, G. R., Stosic, T., \& Stosic, B. (2021). Correlações em séries temporais de preços de frango, soja e milho. Research, Society and Development, 10(4), e20610414019-e20610414019.

Podobnik, B., \& Stanley, H. E. (2008). Detrended cross-correlation analysis: a new method for analyzing two nonstationary time series. Physical review letters, $100(8), 084102$.

Shin, K. H., Lim, G., \& Min, S. (2020). Dynamics of the Global Stock Market Networks Generated by DCCA Methodology. Applied Sciences, $10(6), 2171$.

Siqueira Jr, E. L., Stošić, T., Bejan, L., \& Stošić, B. (2010). Correlations and cross-correlations in the Brazilian agrarian commodities and stocks. Physica A: Statistical Mechanics and its Applications, 389(14), 2739-2743.

Souza, J. D. S., de Almeida, C. O., Araújo, J. L. P., \& Cardoso, C. E. L. (2002). Aspectos socioeconômicos. Embrapa Semiárido-Capítulo em livro técnico (INFOTECA-E).

Souza, S., Pereira, A. F. C., de Lima, J. R. F., \& de Souza, A. L. (2018). Transmissão de preços da manga brasileira entre os mercados interno, americano e europeu. In Embrapa Semiárido-Artigo em anais de congresso (ALICE). In: Congresso Da Sociedade Brasileira De Economia, Administração E Sociologia Rural, 56., 2018, Campinas. Transformação recentes na agropecuária brasileira: desafios em gestão, inovação, sustentabilidade e inclusão social. Campinas: UNICAMP, 2018.

Stosic, T., Telesca, L., da Costa, S. L. L., \& Stosic, B. (2016). Identifying drought-induced correlations in the satellite time series of hot pixels recorded in the Brazilian Amazon by means of the detrended fluctuation analysis. Physica A: Statistical Mechanics and its Applications, 444, 660-666. 
Research, Society and Development, v. 10, n. 12, e266101220460, 2021

(CC BY 4.0) | ISSN 2525-3409 | DOI: http://dx.doi.org/10.33448/rsd-v10i12.20460

Thaxton, C. S., Anderson, W. P., Gu, C., Stosic, B., \& Stosic, T. (2018). Detrended fluctuation analysis and entropy-complexity causality analysis of temperatures in an urbanized mountain stream. Stochastic environmental research and risk assessment, 32(3), 843-858.

Valadares, G. M., \& Landau, E. C. (2020). Evolução da produção de manga (Mangifera indica, Anacardiaceae). Embrapa Milho e Sorgo-Capítulo em livro científico (ALICE).

Vassoler, R. T., \& Zebende, G. F. (2012). DCCA cross-correlation coefficient apply in time series of air temperature and air relative humidity. Physica A: Statistical Mechanics and its Applications, 391(7), 2438-2443.

Willson, K., Francis, D. P., Wensel, R., Coats, A. J., \& Parker, K. H. (2002). Relationship between detrended fluctuation analysis and spectral analysis of heartrate variability. Physiological measurement, 23(2), 385.

Zebende, G. F. (2011). DCCA cross-correlation coefficient: Quantifying level of cross-correlation. Physica A: Statistical Mechanics and its Applications, 390(4), 614-618.

Zebende, G. F., Da Silva, M. F., \& Machado Filho, A. (2013). DCCA cross-correlation coefficient differentiation: Theoretical and practical approaches Physica A: Statistical Mechanics and its Applications, 392(8), 1756-1761.

Zhao, J. C., Dou, W. H., Ji, H. D., \& Wang, J. (2013). Detrended cross-correlation analysis of epilepsy electroencephalagram signals. In Advanced Materials Research (Vol. 765, pp. 2664-2667). Trans Tech Publications Ltd.

Zheng, Z., Yamasaki, K., Tenenbaum, J., Podobnik, B., Tamura, Y., \& Stanley, H. E. (2012). Scaling of seismic memory with earthquake size. Physical Review E, 86(1), 011107. 